\title{
Application of Quantum-Chemical Methods Including Density Functional Theory for the Interpretation of Isotropic Hyperfine Data. The Example of Azulenebenzoquinone
}

\author{
G. Gescheidt ${ }^{1}$, F. Barbosa ${ }^{2}$, J. Daub ${ }^{3}$, and J. Bindl ${ }^{3}$ \\ ${ }^{1}$ Institute of Physical Chemistry, University of Basel, Basel, Switzerland \\ ${ }^{2}$ Institute of Organic Chemistry, University of Basel, Basel, Switzerland \\ ${ }^{3}$ Institute of Organic Chemistry, University of Regensburg, Regensburg, Germany
}

Received June 23, 1997; revised July 23, 1997

Dedicated to Harry Kurreck on the occasion of his 65 th birthday

\begin{abstract}
The radical anion of aceneazulenedione in which a benzoquinone is fused to an azulene moiety was generated by electrolysis and by reduction with the alkali metals in ethereal solvents. The hyperfine data could not be reproduced by standard Hückel calculations which usually give reliable predictions for the spin distribution in radical ions such as azulene quinones and a variety of extended $\pi$ systems. However, PPP and, preferably, ab initio geometry optimisations followed by single-point calculations of the Fermi contact interaction with density functional theory, led to a straightforward assignment of the hyperfine coupling constants.
\end{abstract}

\section{Introduction}

Molecules being composed of donor and acceptor moieties are of interest as rechargeable optoelectronic devices in material science. Even in nature many processes are guided by interactions between donor and acceptor domains. One of the most prominent examples is photosynthesis where the electron transfer takes place between a porphyrine donor and a benzoquinone acceptor.

One-electron reduction is the predominant reaction pathway of the acceptor moieties. ESR spectroscopy including the multiple resonance techniques like ENDOR and TRIPLE are the methods of choice to establish the structure of the radical anions formed as the primary intermediates [1]. Comparison of the experimental hyperfine data with their theoretical counterparts often leads to an unambiguous identification of the geometry and structure of the species giving rise to the ESR signal. 


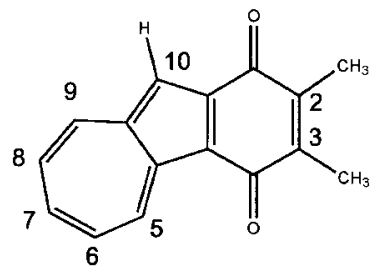

Fig. 1. Compound 1.

A vast palette of quantum chemical methods have been utilised for the calculation of isotropic hyperfine coupling constants. As far as delocalised $\pi$ systems are regarded, the Hückel $\mathrm{MO}$ method, including various sets of perturbation parameters [2], and rarely the Pariser-Parr-Pople (PPP-SCF) [3] method, have been applied with considerable success.

For systems in which the electron-transfer reaction leading to the desired radical induces particular changes of the molecular geometry, the predictive quality of these two methods decreases significantly.

The application of density functional theory (DFT) $[4,5]$ for the calculation of the Fermi contact interaction was shown to be rather efficient. However, mostly small systems have been studied hitherto [6-9]. In line with the findings by Gauld, Eriksson and Radom [7] we have shown that the three-parameter hybrid exchange functional with HF (Hartree-Fock) suggested by Becke [10] using the correlation functional by Lee, Yang and Parr [11], B3LYP, is a useful method for the calculation of hyperfine coupling constants of large systems [12]. Moreover it was found that geometry optimisations can be favorably performed with the $a b$ initio Hartree-Fock method using the $3-21 \mathrm{G}^{*}$ basis set because of convenient CPU times when the Gaussian [13] program package is used [12].

Only rarely has DFT been applied to calculate the hyperfine coupling constants of extended organic $\pi$ systems [14] and, to our knowledge, DFT has not yet been tested for its applicability for non-alternating $\pi$ systems.

The aim of this contribution is to inspect if the combination of ab initio and DFT procedures helps to gain insight into the radical anion of molecule 1 which consists of a 1,4-benzoquinone acceptor fused to a (non-alternating) azulene moiety (Fig. 1).

\section{Results}

It has been established that $\mathbf{1}$ is a very efficient electron acceptor [15-18]. The reduction potential for the formation of the radical anion $1^{--}$is -0.61 vs. SCE (solvent, DMF; supporting salt, tetrabutylammoniumperchlorate; working electrode, platinum disk). 


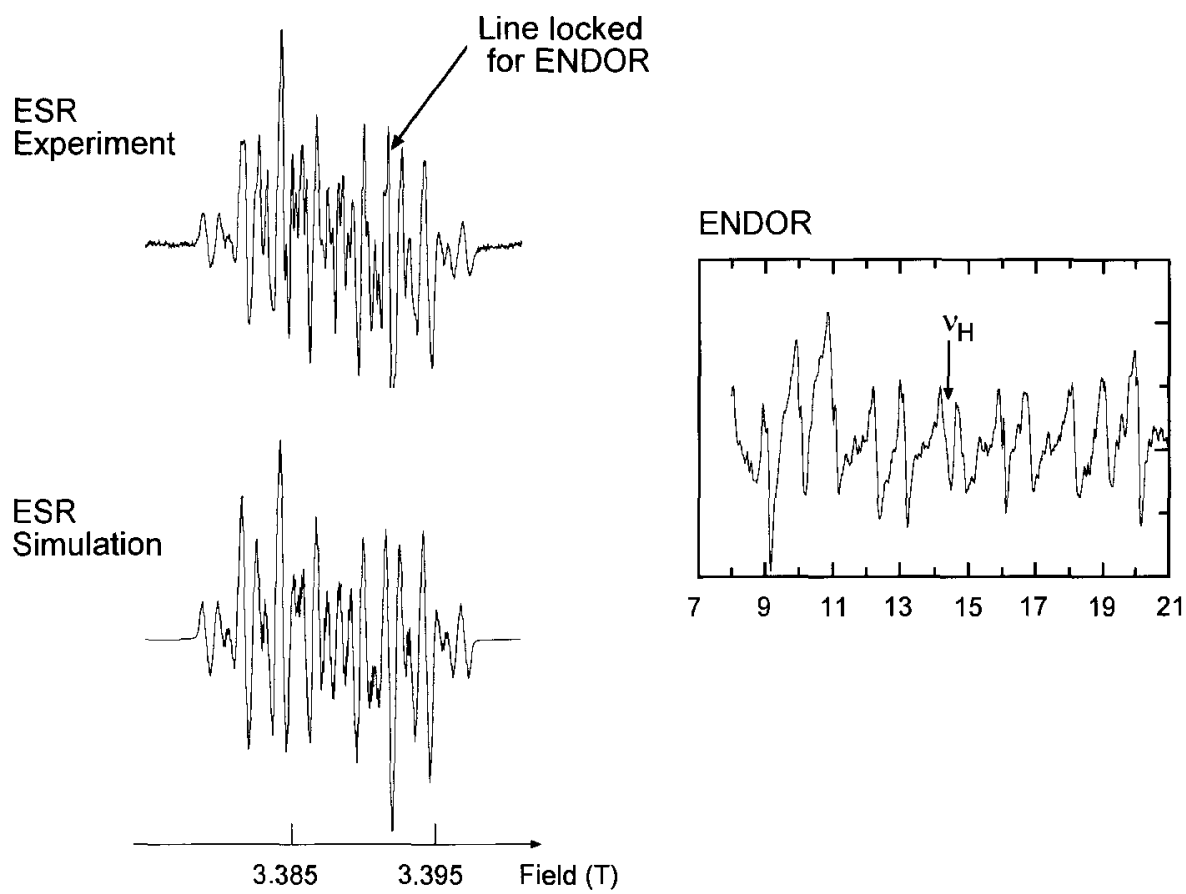

Fig. 2. ESR spectrum of $1^{\cdot-}$ (top) and its computer simulation (bottom). The inset shows the corresponding ENDOR spectrum (temperature: $243 \mathrm{~K}$; solvent DME; counterion $\mathrm{K}^{+}$); $a_{\mathrm{H}}(\mathrm{mT})=0.261$ $(3 \mathrm{H}), 0.014(3 \mathrm{H}), 0.392(1 \mathrm{H}), 0.329(1 \mathrm{H}), 0.165(1 \mathrm{H}), 0.015(1 \mathrm{H}), 0.027(1 \mathrm{H}) ; g=2.00409$; microwave power for ESR: $2 \mathrm{~mW}$, for ENDOR: $12 \mathrm{~mW}$.

Owing to their thermodynamical stability rather persistent radical anions of 1 could be generated by in situ electrolysis [19] or metal reduction $(\mathrm{K})$ in 1,2dimethoxyethane (DME), and mixtures of DME with $\mathrm{N}, \mathrm{N}, \mathrm{N}^{\prime}, \mathrm{N}^{\prime}, \mathrm{N}^{\prime \prime}, \mathrm{N}^{\prime \prime}$-hexamethylphosphoricacid triamide (HMPA). Figure 2 shows the ESR spectrum of $1^{--}$in DME with $\mathrm{K}^{+}$as the counterion as well as its computer simulation. The analysis of the ESR spectra and their simulation was only rendered possible with the help of the ENDOR and general TRIPLE techniques [1].

In Table 1 the experimental hyperfine coupling constants of $\mathbf{1}^{--}$, formed by $\mathrm{K}$ metal reduction in a mixture of DME/HMPA 5:1 are compared to their calculated counterparts.

To assign of the experimental $a_{\mathrm{H}}$ to individual positions in $1^{*}$, quantum mechanical calculations at different levels of theory were performed. The regularly applied procedure for the prediction of $a_{\mathrm{H}}$ of radical ions of delocalised $\pi$ systems is to use the Hückel model (HMO). Consequently, the $a_{\mathrm{H}}$ of $1^{--}$were determined by the HMO-McLachlan [20] procedure. The Pariser-Parr-Pople (PPP) [21] method, like HMO only takes into account the $\pi$ orbitals, and has also been successfully used for the calculation of hyperfine coupling constants [3]. Recent 
Table 1. Experimental $a_{\mathrm{H}}$ of 1- and the corresponding calculated values by Hückel MO, PPP, and $\mathrm{DFT} / a b$ initio.

\begin{tabular}{ccccc}
\hline Position $(3 \mathrm{H})$ & $a_{\mathrm{H}} \exp ^{\mathrm{a}}(\mathrm{mT})$ & $a_{\mathrm{H}} \mathrm{HMO}^{\mathrm{a}}(\mathrm{mT})$ & $a_{\mathrm{H}} \mathrm{PPP}^{\mathrm{b}}(\mathrm{mT})$ & $a_{\mathrm{H}} \mathrm{DFT}^{\mathrm{c}}(\mathrm{mT})$ \\
\hline 2 & +0.034 & -0.12 & -0.092 & +0.045 \\
3 & +0.202 & -0.31 & -0.138 & +0.202 \\
5 & -0.436 & -0.15 & -0.376 & -0.415 \\
6 & +0.113 & -0.02 & -0.001 & +0.164 \\
7 & -0.361 & -0.14 & -0.350 & -0.359 \\
8 & +0.013 & -0.04 & -0.074 & +0.019 \\
9 & -0.170 & -0.12 & -0.222 & -0.180 \\
10 & +0.013 & -0.21 & -0.054 & +0.025 \\
\hline
\end{tabular}

a Values according to Hückel-McLachlan procedure [20] $(\lambda=1.2)$, perturbation parameters for the quinone $O$ atoms: $\alpha_{\mathrm{O}}=\alpha+2 \beta, \beta_{\mathrm{CO}}=1.5 \beta$.

${ }^{b}$ Based on PPP-SCF calculations, conversion factor $Q=-2.7 \mathrm{mT}$.

${ }^{c}$ UB3LYP/6-31G"/UHF/3-21G*.

studies indicate that the calculation of the Fermi contact interaction, being responsible for the $a_{\mathrm{H}}$, with the use of density functional methods (DFT) often reveals a very good agreement with the experimental data $[9,12,14,22]$. Therefore the geometry of $1^{-}$was determined by $a b$ initio calculations at the unrestricted Hartree-Fock level of theory. With this geometry a single point calculation with the Becke [10] three parameter hybrid method with HF using the correlation functionals of Lee, Yang and Parr [11] and the local correlation functional by Vosko, Wilk and Nusair [23] (B3LYP) was performed. The results of the calculations are compared with the experimental data in Table 1.

\section{Discussion}

The Hückel model has been shown to offer valuable predictions for hyperfine coupling constants of radical ions derived from $\pi$ systems [2] and although no geometrical constraints are accounted for by the graph-type cognition of struc-

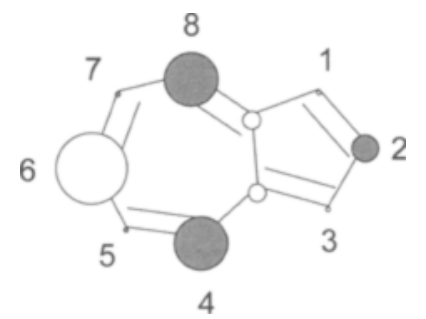

Fig. 3. LUMO of azulene according to the Hückel model; the same shape is also indicated by PPP and $a b$ initio/DFT calculations. 
tures rather complicated systems have been described with this model [24-26]. It is therefore not astonishing that the azulene radical anion has been described in terms of its HMO derived lowest unoccupied molecular orbital (LUMO). The experimental data are in line with the prominent hyperfine coupling constants stemming from protons (or methyl groups) at the positions with even numbering (Fig. 3) [27]. The same shape of the azulene LUMO is also corroborated by PPP or DFT calculations.

But, when the benzoquinone moiety is fused to the azulene yielding the parent compound 1, the HMO prediction for the corresponding LUMO indicates a shape in which the spin and the charge are predominately localised in the quinone moiety (Fig. 4). Such a spin distribution can not be correlated to the experimental $a_{\mathrm{H}}$ established for $\mathbf{1}^{*-}$. From the simulation of the ESR spectra it can clearly be re-

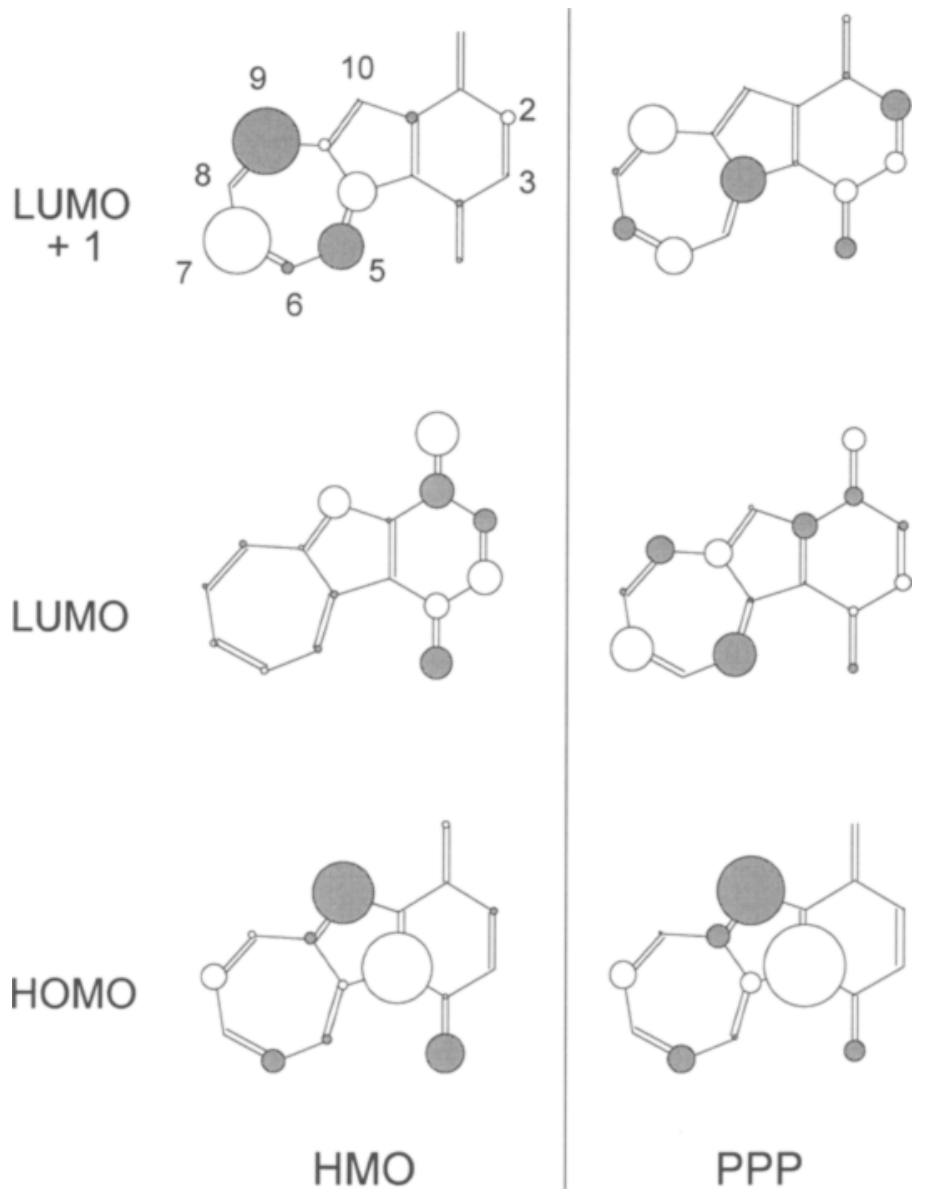

Fíg. 4. Frontier orbitals of 1 (HOMO, LUMO and LUMO +1 according to Hückel (left) and PPP (right) calculations. The shape of the LUMO indicated by PPP is in accord with the UB3LYP/ $6-31 \mathrm{G}^{*} / \mathrm{UHF} / 3-21 \mathrm{G}^{*}$ calculated $a_{\mathrm{H}}$. 
solved that the three-proton $a_{\mathrm{H}}$ of the methyl groups in the 2,3 positions of 1 have distinctly differing values (by one order of magnitude). In contrast, the HMOLUMO of 1 (Fig. 4, left) these coefficients differ by the factor of ca. 2.5. Moreover, no coefficient of considerable size exists in the seven membered ring and the only position which carries a conspicuous $a_{\mathrm{H}}$ is that at $\mathrm{C}(10)$ in the five ring of the azulene moiety. Such an electron distribution does not correlate with the experimental observations (Table 1). On the Hückel level, the LUMO +1 (Fig. 4) indicates a fair agreement with the experimental $a_{\mathrm{H}}$. The major coefficients are localised in the azulene moiety and the spin population at $\mathrm{C}(10)$ is almost zero. It is, however unlikely that an alkali-metal reduction of 1 immediately leads to the occurrence of a radical trianion which is represented by the LUMO +1 .

Application of the PPP model [21] which is a self-consistent-field (SCF) procedure brings about a distinctly different type of LUMO. The electron is distributed among both the azulene and the quinone constituents of $1^{\circ-}$. The spin population in the azulene moiety closely resembles that of the parent unsubstituted azulene (Fig. 4, right, compare to Fig. 3). Indeed the $a_{\mathrm{H}}$ derived from the PPP calculated LUMO with the conversion factor $Q=2.7 \mathrm{mT}$ [3] indicate a satisfactory agreement with the experimental data (Table 1). It is noteworthy that this PPP LUMO is very similar to the LUMO +1 in the Hückel model (Fig. 4) whereas the HOMOs have identical shapes.

The two related methods Hückel and PPP give distinctly differing descriptions for the $a_{\mathrm{H}}$ of $1^{--}$, therefore a third procedure which has been shown to give rather reliable predictions of hyperfine coupling constants in several classes of radicals and for different atoms was utilised [12]. The $a_{\mathrm{H}}$ obtained the combination of $a b$ initio (Hartree-Fock) and DFT (B3LYP) are in very good agreement with the experimental data (Table 1) and closely correlate with the shape of the PPP-calculated LUMO (Fig. 4). The geometry of $1^{--}$calculated by UHF/3-21G* is shown in Fig. 5. The lengths of the benzoquinone $\mathrm{C}=\mathrm{O}$ are ca. $1.27 \AA$ long. For a semiquinone radical anion a significant shortening of the $\mathrm{C}=\mathrm{O}$ bond (ca. 1.35 $\AA$ in the neutral molecule) would be expected [28]. The second particularity is

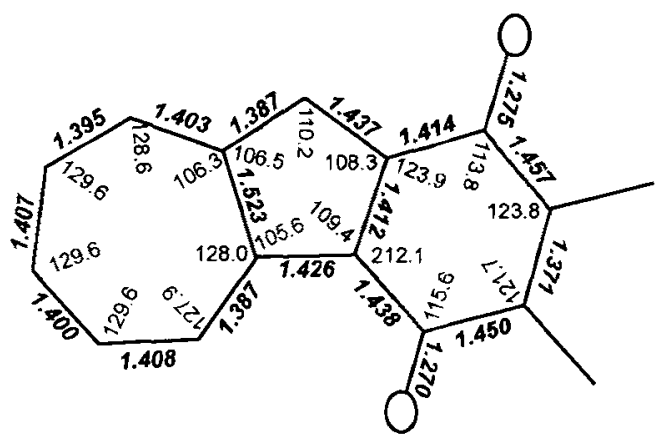

Fig. 5. Bond lengths ( $\AA$, bold-italic) and bond angles (in degrees) of $1^{--}$according to UHF/3-21G calculation. 


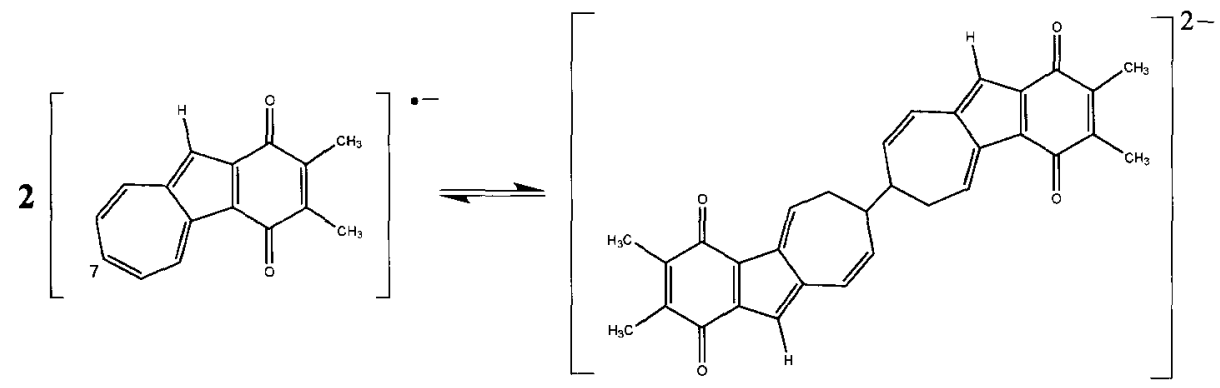

Fig. 6. Dimerization of $\mathbf{1}$ after one-electron reduction according to cyclovoltammetric measurements.

the bond connecting the seven and the five ring of the azulene moiety which amounts to $1.52 \AA$, i.e., a formal $\mathrm{C}-\mathrm{C}$ single bond.

Thus the failure of the Hückel model in the case of $1^{--}$can be traced back to the non standard bond length of $1^{--}$exhibited by the $a b$ initio calculated geometry. Moreover the typical perturbation parameters of the Hückel model overestimate the electronegativity of the $\mathrm{O}$ atoms and presumably underestimate the inductive effect of the methyl groups. Consequently, following the Hückel calculation the entire negative charge resides at the benzoquinone part of $1^{--}$whereas the azulene moiety possesses only positive charges. According to the $a b$ initio structure, the azulene atoms $\mathrm{C}(5)-\mathrm{C}(10)$ carry negative charges. This behaviour verifies that caution has to be taken when heteroatoms or substituents are modelled by perturbation parameters in the Hückel treatment.

The shape of the LUMO with the highest amount of spin population residing at the $C(5)$ and $C(7)$ of $\mathbf{1}^{--}$corresponds favorably with the reactivity established for 1 by cyclovoltammetric measurements. After the first electron transfer, the radical anion formed tends to undergo a dimerisation reaction [29]; the formation of the new bond occurs at the seven membered ring of the azulene moiety where position $C(7)$ seems to be the preferred position (Fig. 6).

In this respect, further studies involving more derivatives of 1 seems worthwhile. Moreover, it will be interesting to inspect in how far ion-pairing effects can influence the structure and reactivity of azulene containing electron acceptors.

\section{Experimental}

ESR spectra were recorded on a Varian E9 or a Bruker ESP 300 spectrometer. ENDOR measurements were performed on a Varian E9 instrument equipped with an ENDOR unit or on the Bruker ESP 300. The latter instrument was also used for the general TRIPLE technique. The field frequency lock for the ENDOR spectra was set at the most intense high field line of the ESR signals. For the TRIPLE measurements, typically three different lines of the ENDOR spectrum 
were pumped to achieve an unambiguous assignment of the relative signs of the $a_{\mathrm{H}}$. For the determination of the absolute sign, the major $a_{\mathrm{H}}$ stemming from the 5 and 7 positions of the seven membered ring were taken as negative (in line with the predictions of the DFT calculations). ESR spectra were simulated with the program Winsim [30].

The syntheses of the aceneazulenediones were described in $[18,31]$.

Hückel calculations were done with the program MacHMO [32] and PPP was performed with the program PPPinput/PPPcalc [33]. The ab initio and DFT calculations were performed with Gaussian 94 [13] using a modified treatment of the B3LYP hybrid functional [34]. The HF and the DFT calculations were performed at the unrestricted level of theory.

\section{Acknowledgements}

We are indebted to Dr. Rohit Batra (Basel) for his help with the calculations. F.B. thanks Prof. Bernd Giese for support.

\section{References}

[1] Kurreck H., Kirste B., Lubitz W.: Electron Nuclear Double Resonance Spectroscopy of Radicals in Solution. Weinheim: VCH 1988.

[2] Gerson F.: Hochauflösende ESR-Spektroskopie. Weinheim: Verlag Chemie 1967.

[3] Liberko C.A., Rak S.F., Miller L.L.: J. Org. Chem. 57, 1379 (1992)

[4] Parr R.G., Yang W.: Density Functional Theory of Atoms and Molecules. Oxford: Oxford University Press 1989.

[5] Kohn W., Becke A.D., Parr A.G.: J. Phys. Chem. 100, 12974 (1996)

[6] Malkin V.G., Malkina O.L., Eriksson L.A., Salahub D.R. in: Theoretical and Computational Chemistry (Seminario J.M., Politzer P., eds.), vol. 2. Amsterdam: Elsevier 1995.

[7] Gauld J.W., Eriksson L.A., Radom L.: J. Phys. Chem. A 101, 1352 (1997)

[8] Suter H.U., Pless V., Ernzerhof M., Engels B.: Chem. Phys. Lett. 230, 398 (1994)

[9] Eriksson L.A., Laaksonen A.: J. Chem. Phys. 105, 8195 (1996)

[10] Becke A.D.: J. Chem. Phys. 98, 1372 (1993)

[11] Lee C., Yang W., Parr R.G.: Phys. Rev. B 37, 785 (1988)

[12] Batra R., Giese B., Spichty M., Gescheidt G., Houk K.N.: J. Phys. Chem. 100, 18371 (1996)

[13] Frisch M.J., Trucks G.W., Schlegel H.B., Gill P.M.W., Johnson B.G., Robb M.A., Cheeseman J.R., Keith T., Petersson G.A., Montgomery J.A., Raghavachari K., Al-Laham M.A., Zakrzewski V.G., Ortiz J.V., Foresman J.B., Cioslowski J., Stefanov B.B., Nanayakkara A., Challacombe M., Peng C.Y., Ayala P.Y., Chen W., Wong M.W., Andres J.L., Replogle E.S., Gomperts R., Martin R.L., Fox D.J., Binkley J.S., Defrees D.J., Bake J., Stewart J.P., Head-Gordon M., Gonzalez C., Pople J.A.: Gaussian 94, Revision B.2. Pittsburgh PA 1995.

[14] Gano J.E., Jacob E.J., Sekher P., Subramaniam G., Eriksson L.A., Lenoir D.: J. Org. Chem. 61, 6739 (1996)

[15] Daub J., Salbeck J., Knöchel T., Fischer C., Kunkel H., Rapp K.M.: Angew. Chem. 101, 1541 (1989)

[16] Gierisch S., Bauer W., Burgemeister T., Daub J.: Chem. Ber. 122, 2341 (1989)

[17] Daub J., Rapp K.M., Salbeck J., Schöberl U. in: Carbohydrates as Organic Raw Materials (Lichtenthaler W., ed.), pp 323. Weinheim New York: VCH 1991.

[18] Bindl J., Daub J., Hasenhuendl A., Meinert M., Rapp K.M.: Chem. Ber. 116, 2408 (1983) 
[19] Ohya-Nishiguchi H.: Bull. Chem. Soc. Jpn. 52, 2064 (1979)

[20] McLachlan A.D.: Mol. Phys. 3, 233 (1960)

[21] Pariser R., Parr R.G.: J. Chem. Phys. 21, 466 (1953)

[22] Barone V., Adamo C., Grand A., Jolibois F., Brunel Y., Subra R.: J. Am. Chem. Soc. 117, 12618 (1995)

[23] Vosko S.H., Wilk R., Nusair M.: Can. J. Phys. 58, 1200 (1980)

[24] Bachmann R., Gerson F., Gescheidt G., Hafner K.: Magn. Reson. Chem. 33, 60 (1995)

[25] Bachmann R., Gerson F., Merstetter P., Vogel E.: Helv. Chim. Acta 79, 1627 (1996)

[26] Gerson F., Lamprecht A., Scholz M., Lenoir D.: Helv. Chim. Acta 79, 307 (1996)

[27] Bachmann R., Burda C., Gerson F., Scholz M., Hansen J.: Helv. Chim. Acta 77, 1458 (1994)

[28] Shinagawa Y., Shinagawa Y.: J. Am. Chem. Soc. 100, 67 (1978)

[29] Daub J., Salbeck J.: Chem. Ber. 122, 727 (1989)

[30] Duling D.: P.E.S.T. WinSim, NIH, Research Triangle Park 1996.

[31] Bindl J.: Doctoral Thesis, University of Regensburg, Regensburg 1985.

[32] Huber H.: MacHMO, University of Basel, Basel 1985.

[33] Naef R.: PPPinput/PPPcalc, Basel 1993.

[34] Stephens P.J., Devlin J.F., Chabalowski F., Frisch M.J.: J. Phys. Chem. 98, 11623 (1994)

Author's address: Dr. Georg Gescheidt, Institute of Physical Chemistry, University of Basel, Klingelbergstrasse 80, 4056 Basel, Switzerland 